PROCEEDINGS OF THE

AMERICAN MATHEMATICAL SOCIETY

Volume 126, Number 10, October 1998, Pages 2927-2932

S $0002-9939(98) 04411-6$

\title{
SAMPLING SETS FOR HARDY SPACES OF THE DISK
}

\author{
PASCAL J. THOMAS
}

(Communicated by Theodore W. Gamelin)

\begin{abstract}
We propose two possible definitions for the notion of a sampling sequence (or set) for Hardy spaces of the disk. The first one is inspired by recent work of Bruna, Nicolau, and Øyma about interpolating sequences in the same spaces, and it yields sampling sets which do not depend on the value of $p$ and correspond to the result proved for bounded functions $(p=\infty)$ by Brown, Shields and Zeller. The second notion, while formally closer to the one used for weighted Bergman spaces, leads to trivial situations only, but raises a possibly interesting problem.
\end{abstract}

\section{§1. Sampling via the Bruna-Nicolau-Øyma function}

Let $\mathbb{D}$ be the unit disk in the complex plane. Recall that for any $0<p \leq \infty$ the Hardy space $H^{p}(\mathbb{D})$ is the set of holomorphic functions $f$ such that

$$
\|f\|_{p}:=\left(\sup _{r<1} \int_{0}^{2 \pi}\left|f\left(r e^{i \theta}\right)\right|^{p} \frac{d \theta}{2 \pi}\right)^{\frac{1}{p}}<\infty,
$$

where the integral is replaced by a supremum in the case $p=\infty$, and that for $p \geq 1$, $\|\cdot\|_{p}$ is a norm.

In accordance with many previous works (e.g. [La], [Se1], [Se2]) we would like to say that a subset $a$ of the unit disk is of sampling for the space $H^{p}(\mathbb{D})$ when the values of a function $f \in H^{p}(\mathbb{D})$, restricted to this set, determine the function uniquely, and when we can establish some inequalities between the $H^{p}$-norm and an appropriate norm on the space of functions on the subset $a$. Usually, this is interesting only when the subset $a$ is a discrete sequence of points, however, that hypothesis will not be necessary for the first part of this paper. We proceed to give a more specific definition.

The Stolz angle with vertex at $e^{i \theta}$ and aperture $\alpha>0$ is

$$
\Gamma_{\alpha}\left(e^{i \theta}\right):=\left\{z \in \mathbb{D}: \frac{\left|e^{i \theta}-z\right|}{1-|z|}<1+\alpha\right\} .
$$

The nontangential maximal function is

$$
M f\left(e^{i \theta}\right):=\sup _{z \in \Gamma_{\alpha}\left(e^{i \theta}\right)}|f(z)| .
$$

Received by the editors October 14, 1996 and, in revised form, February 27, 1997. 1991 Mathematics Subject Classification. Primary 30E10, 30D55, 30C15.

(C)1998 American Mathematical Society 
For any $0<p \leq \infty$, for any choice of $\alpha>0$, we have $\|M f\|_{p} \leq C_{p, \alpha}\|f\|_{p}$, where, for functions defined on the unit circle, $\|\cdot\|_{p}$ stands for the usual norm in the space $L^{p}\left(\frac{d \theta}{2 \pi}\right)$ ([Du], [Ga, Theorem II.3.1, p. 57]).

Now, following Bruna-Nicolau-Øyma [Br-Ni-Øy], let

$$
M_{a}(f)\left(e^{i \theta}\right):=M_{a, \infty}(f)\left(e^{i \theta}\right):=\sup _{\Gamma_{\alpha}\left(e^{i \theta}\right) \cap a}|f| \leq M f\left(e^{i \theta}\right) .
$$

We write $M^{\alpha} f, M_{a}^{\alpha} f$ when we want to denote dependence on the aperture. From the above it follows that $\left\|M_{a}^{\alpha}(f)\right\|_{p} \leq C_{p, \alpha}\|f\|_{p}$. We will call the set $a$ sampling if the two norms are actually equivalent.

Definition 1. We say that the set $a$ is sampling for $H^{p}(\mathbb{D})$ iff there exists a constant $\alpha>0$ and a constant $C>0$ such that for any $f \in H^{p}(\mathbb{D}),\left\|M_{a}^{\alpha}(f)\right\|_{p} \geq C\|f\|_{p}$.

In the case where $p=\infty$, this simply says that $\sup _{a}|f| \geq C \sup _{\mathbb{D}}|f|$, and by taking powers of $f$ we see that $\sup _{a}|f|=\sup _{\mathbb{D}}|f|$. This case of the problem was solved by Brown, Shields and Zeller [Br-Sh-Ze, Th. 3, (iii)-(iv)]. The main positive result of this note is that, with the appropriate definition above, this can be extended to all $p>0$.

Definition. We write $e^{i \theta} \in N T_{\alpha}(a)$ iff $e^{i \theta}$ is in the closure of $\Gamma_{\alpha}\left(e^{i \theta}\right) \cap a$. We say that a point of the circle $e^{i \theta}$ is a nontangential limit point of the set $a$ iff $e^{i \theta} \in N T(a):=\bigcup_{\alpha>0} N T_{\alpha}(a)$.

Denote by $\lambda_{1}$ the 1-dimensional Lebesgue measure on the unit circle.

Theorem 1. The set $a$ is sampling for $H^{p}(\mathbb{D})$ if and only if $\lambda_{1}$-almost every point $e^{i \theta} \in \partial \mathbb{D}$ is a nontangential limit point of the set a, i.e. $\lambda_{1}(\partial \mathbb{D} \backslash N T(a))=0$.

Remark. Observe that the condition in the theorem is equivalent to the seemingly stronger one that there be some $\alpha>0$ such that $\lambda_{1}\left(\partial \mathbb{D} \backslash N T_{\alpha}(a)\right)=0$. This follows from the fact that for any $\alpha<\beta, N T_{\alpha}(a) \subset N T_{\beta}(a)$ and $\lambda_{1}\left(N T_{\beta}(a) \backslash N T_{\alpha}(a)\right)=0$.

To prove this last assertion, set $E_{\alpha}:=\partial \mathbb{D} \backslash N T_{\alpha}(a)$. Then

$$
E_{\alpha}=\bigcup_{N>0}\left\{e^{i \theta} \in \partial \mathbb{D}: a \cap \Gamma_{\alpha}\left(e^{i \theta}\right) \backslash D\left(0,1-\frac{1}{N}\right)=\emptyset\right\}=: \bigcup_{N>0} E_{\alpha, N} .
$$

Referring to [St, (3), p. 202] we see that for any $\varepsilon>0$ there exists $N(\varepsilon)>N$ and $E_{\alpha, N}^{(\varepsilon)} \subset E_{\alpha, N}$ such that $\lambda_{1}\left(E_{\alpha, N} \backslash E_{\alpha, N}^{(\varepsilon)}\right)<\varepsilon$, and

$$
\bigcup_{e^{i \theta} \in E_{\alpha, N}^{(\varepsilon)}}\left(\Gamma_{\beta}\left(e^{i \theta}\right) \backslash D\left(0,1-\frac{1}{N(\varepsilon)}\right)\right) \subset \bigcup_{e^{i \theta} \in E_{\alpha, N}}\left(\Gamma_{\alpha}\left(e^{i \theta}\right) \backslash D\left(0,1-\frac{1}{N}\right)\right),
$$

which is itself contained in $\mathbb{D} \backslash a$ by construction. Thus $E_{\alpha, N}^{(\varepsilon)} \subset \partial \mathbb{D} \backslash N T_{\beta}(a)=: E_{\beta}$ for any $\varepsilon>0$, and

$$
\lambda_{1}\left(E_{\alpha, N} \backslash \bigcup_{M} E_{\alpha, N}^{(1 / M)}\right)=0,
$$

so $\lambda_{1}\left(E_{\alpha} \backslash E_{\beta}\right)=0$. q.e.d.

Proof of Theorem 1. This proof is essentially the same as in [Br-Sh-Ze], so we shall keep it brief. We say that a function $f$ defined on the disk admits a nontangential limit at the point $e^{i \theta}$ iff $\lim _{z \rightarrow e^{i \theta}, z \in \Gamma_{\alpha}\left(e^{i \theta}\right)} f(z)=: f^{*}\left(e^{i \theta}\right)$ exists and is finite. For 
any choice of $\alpha$, any $f \in H^{p}(\mathbb{D})$ admits a nontangential limit at almost every $e^{i \theta}$ and $\left\|f^{*}\right\|_{p}=\|f\|_{p}[\mathrm{Du}]$, [Ga].

Thus for almost every $e^{i \theta} \in N T_{\alpha}(a)$,

$$
M_{a}(f) \geq \lim _{z \rightarrow e^{i \theta}, z \in \Gamma_{\alpha}\left(e^{i \theta}\right) \cap a}|f(z)|=\left|f^{*}\left(e^{i \theta}\right)\right|,
$$

so that if $\lambda_{1}\left(\partial \mathbb{D} \backslash N T_{\alpha}(a)\right)=0,\left\|M_{a}(f)\right\|_{p} \geq\left\|f^{*}\right\|_{p}$. q.e.d.

Conversely, if $\lambda_{1}\left(\partial \mathbb{D} \backslash N T_{\alpha}(a)\right)>0$, there is an integer $N$ and $A$, a compact set of positive measure, such that for all $e^{i \theta} \in A, \Gamma_{\alpha}\left(e^{i \theta}\right) \cap a \cap\{|z| \geq 1-1 / N\}=\emptyset$. Consider the outer function

$$
\omega_{A}(z):=\exp \left\{-\int_{0}^{2 \pi} \frac{z+e^{i \theta}}{z-e^{i \theta}}\left(1-1_{A}\left(e^{i \theta}\right)\right) \frac{d \theta}{2 \pi}\right\}
$$

where $1_{A}$ is the indicator function of $A$. Then $-\log \left|\omega_{A}\right|$ is the harmonic measure of the set $\partial \mathbb{D} \backslash A$, and classical estimates (e.g. [Ga, ex. 3, p. 41]) show that for all $z \notin \bigcup_{e^{i \theta} \in A} \Gamma_{\alpha}\left(e^{i \theta}\right),-\log \left|\omega_{A}\right| \geq c_{\alpha}>0$.

Consider the sequence of functions $f_{n}(z):=z^{n} \omega_{A}(z)^{n}$ : we have $\left\|f_{n}\right\|_{p}^{p}=\left\|f_{n}^{*}\right\|_{p}^{p}$ $\geq \frac{\lambda_{1}(A)}{2 \pi}>0$, while $M_{a}\left(f_{n}\right)\left(e^{i \theta}\right) \leq 1$ for all $n$, and $\lim _{n \rightarrow \infty} M_{a}\left(f_{n}\right)\left(e^{i \theta}\right)=0$ for all $e^{i \theta} \in A$ because $(1-1 / N)^{n} \rightarrow 0$, and for all $e^{i \theta} \notin A$ because $\exp \left(-n c_{\alpha}\right) \rightarrow 0$. So $\left\|M_{a}\left(f_{n}\right)\right\|_{p} \rightarrow 0$, and the sampling inequality cannot hold.

\section{§2. Failure of the "Classical" Definition}

The Bruna-Nicolau-Øyma function was introduced to deal with problems of interpolation of the type studied in [Sh-Sh]. This involved a norm in $L^{p}(\mu)$, where $\mu:=\sum_{z \in a}\left(1-|z|^{2}\right) \delta_{z}$, with $\delta_{z}$ standing for the Dirac mass at the point $z$.

We can then state a similar sampling problem:

Definition 2. We say that the set $a$ is $H^{p}(\mathbb{D})$-thick iff there exists a constant $C>0$ such that for any $f \in H^{p}(\mathbb{D})$,

$$
\|f\|_{L^{p}(\mu)}=\left(\sum_{z \in a}\left(1-|z|^{2}\right)|f(z)|^{p}\right)^{\frac{1}{p}} \geq C\|f\|_{p} .
$$

This says that the measure $\mu$ is dominating in the sense of [Lu].

However, it is a well-known fact that the sum on the left-hand side will then be infinite, which makes for a rather uninteresting notion of sampling.

Proposition 2. The set $a$ is $H^{p}$-thick if and only if $H^{p}(\mathbb{D}) \cap L^{p}(\mu)=\{0\}$ (i.e. if $f \in H^{p}(\mathbb{D})$ and $\sum_{z \in a}\left(1-|z|^{2}\right)|f(z)|^{p}<\infty$, then $\left.f=0\right)$.

Proof. Let $f$ be a function in $L^{p}(\mu) \cap H^{p}(\mathbb{D}) \backslash\{0\}$; set $f_{n}(z):=z^{n} f(z)$. The sequence $\left\{f_{n}\right\}$ is dominated by $|f|$, and we have $\lim _{n \rightarrow \infty} f_{n}(z)=0$ for all $z \in a$. By Lebesgue's dominated convergence theorem in $L^{p}(\mu)$,

$$
\lim _{n \rightarrow \infty} \sum_{z \in a}\left(1-|z|^{2}\right)\left|f_{n}(z)\right|^{p}=0,
$$

while $\left\|f_{n}\right\|_{p}=\|f\|_{p}>0$. So $a$ is not $H^{p}$-thick.

Proposition 3. If $\lambda_{1}(N T(a))>0$, then $H^{p}(\mathbb{D}) \cap L^{p}(\mu)=\{0\}$. 
Proof. Assume $f \in L^{p}(\mu)$. Let us modify the Bruna-Nicolau-Øyma function:

$$
M_{a, p}(f)\left(e^{i \theta}\right):=\left(\sum_{z \in \Gamma_{\alpha}\left(e^{i \theta}\right) \cap a}|f(z)|^{p}\right)^{\frac{1}{p}} \geq M_{a, \infty}(f)\left(e^{i \theta}\right) .
$$

Then

$$
\int_{0}^{2 \pi} M_{a, p}(f)\left(e^{i \theta}\right)^{p} d \theta=\sum_{z \in a}|f(z)|^{p} \int_{\theta: z \in \Gamma_{\alpha}\left(e^{i \theta}\right)} d \theta
$$

and the set $\left\{\theta: z \in \Gamma_{\alpha}\left(e^{i \theta}\right)\right\}$ is an arc centered at $z /|z|$, with length a multiple of $1-|z|$, so the last sum is commensurate to $\|f\|_{L^{p}(\mu)}^{p}$.

So $M_{a, p}^{\alpha}(f)$ must be finite almost everywhere on $\partial \mathbb{D}$. At the points $e^{i \theta} \in N T_{\alpha}(a)$, the sum defining $M_{a, p}^{\alpha}(f)$ is infinite and converges, so

$$
\lim _{z \in \Gamma_{\alpha}\left(e^{i \theta}\right) \cap a, z \rightarrow e^{i \theta}}|f(z)|^{p}=0 .
$$

Suppose now that in addition $f \in H^{p}(\mathbb{D})$. Since $f$ admits a nontangential limit almost everywhere, that limit will be zero almost everywhere on $N T_{\alpha}(a)$. If

$$
\lambda_{1}(N T(a))=\lambda_{1}\left(N T_{\alpha}(a)\right)>0,
$$

then $f=0$ (see e.g. [Ga, Th. 4.1, p. 64]).

In the case of the Bergman spaces studied in [Se1], [Se2], the definition of sampling which is given is akin to Definition 2 (but, unlike it, is not vacuous!). One can also construct an analogue to the first definition, using a supremum on invariant balls of fixed radius rather than Stolz angles. It can be seen, using boundedness of the restriction map which is part of the definition, that the sequences under consideration in [Se1, $\S 7$ ] can only have a bounded number of points in each such ball, which implies that the two notions are in this case equivalent.

The proof uses an inequality exactly analogous to $M_{a, p}(f) \geq M_{a, \infty}(f)$, which shows that if $a$ is sampling for $H^{p}(\mathbb{D})$, then $a$ is $H^{p}(\mathbb{D})$-thick. This comparison could be useful in other situations.

\section{§3. A question, AND AN EXAmple}

Question. Can we characterize explicitly the sets a which are not $H^{p}$-thick, i.e. such that $L^{p}(\mu) \cap H^{p}(\mathbb{D}) \neq\{0\}$ ?

The sets under consideration will have to be discrete sequences, with a nontangential limit set of measure zero. I see the property as a weaker analogue of the Blaschke property (a sequence satisfying the Blaschke property, being a zero-set for an $H^{\infty}$ function, automatically satisfies our condition). It is likely that the methods used by Lyubarskii and Seip in [Ly-Se] to deal with a similar problem will prove useful.

The $H^{p}$-thickness property cannot be about the mere growth of the number of points in the sequence as it approaches the boundary, as is demonstrated by the following example: take $\left\{b_{n}\right\}$ to be a sequence of points in the disk satisfying the Blaschke condition, and let $\left\{b_{n, k}, 1 \leq k \leq q_{n}\right\}$ be $q_{n}$ distinct points in the disk $D\left(b_{n}, q_{n}^{-1}\left(1-\left|b_{n}\right|^{2}\right)^{2}\right)$. Set $a:=\left\{b_{n, k}, 1 \leq n, 1 \leq k \leq q_{n}\right\}$. It is easy to check that the Blaschke product with simple zeroes at each of the $b_{n}$ 's is in $L^{p}(\mu) \cap H^{p}(\mathbb{D})$ for any $p \geq 1$, but of course $q_{n}$ can grow as fast as we please. 
The $H^{p}$-thickness property does not depend on $p$, in keeping with the fact that zero-sets or interpolating sequences for the Hardy spaces $H^{p}(\mathbb{D})$ do not depend on $p$.

Lemma 1. $H^{p}(\mathbb{D}) \cap L^{p}(\mu) \neq\{0\}$ if and only if $H^{\infty}(\mathbb{D}) \cap L^{1}(\mu) \neq\{0\}$.

Proof of Lemma 1. This uses the same ideas as the canonical factorization of functions in the Nevanlinna class. Let $f \in H^{p}(\mathbb{D}) \cap L^{p}(\mu) \backslash\{0\}$. Let $m(z)$ be a harmonic majorant of $|f|^{p}[\mathrm{Ga}$, p. 50]. Since $m(z) \geq 0$, if we denote by $\tilde{m}$ its harmonic conjugate, $1+m(z)+i \tilde{m}(z)=e^{H(z)}$, with $e^{\frac{1}{p} \Re H(z)} \geq \max (1,|f(z)|)$. Then

$$
f_{1}(z):=e^{-\frac{1}{p} H(z)} f(z) \in H^{\infty}(\mathbb{D}), \quad\left\|f_{1}\right\|_{\infty} \leq 1,
$$

and $\left|f_{1}(z)\right| \leq|f(z)|$, so $f_{1} \in L^{p}(\mu)$. If $p \leq 1,\left|f_{1}(z)\right|^{p} \geq\left|f_{1}(z)\right|$ and $f_{1} \in H^{\infty}(\mathbb{D}) \cap$ $L^{1}(\mu)$. If $p>1$, we write $f_{1}(z)=B(z) e^{h(z)}$, where $B$ is a Blaschke product, and set $g(z)=B(z)^{[p]+1} e^{p h(z)}$; then $|g(z)| \leq\left|f_{1}(z)\right|^{p}$, so $g \in H^{\infty}(\mathbb{D}) \cap L^{1}(\mu)$.

The converse implication is left to the reader.

Proposition 3 gives as necessary condition for $H^{p}(\mathbb{D}) \cap L^{p}(\mu) \neq\{0\}$ that $\lambda_{1}(N T(a))$ $=0$. It is however far from sufficient.

Proposition 4. There exists a discrete sequence, accumulating at one point of the circle only, such that $H^{p}(\mathbb{D}) \cap L^{p}(\mu)=\{0\}$.

Proof. Let $\left\{p_{n}\right\}$ be chosen so that $2^{-n} p_{n} \rightarrow \infty$, and take $\gamma_{n}:=2^{n} / 2 p_{n}$. Set $\ell_{n}:=p_{n}^{-1}\left|\log \gamma_{n}\right|^{-1 / 2}$, and

$$
a_{n, k}:=\left(1-2^{-n}\right)^{\frac{1}{2}} \exp i k \ell_{n}, \quad 0 \leq k \leq p_{n} .
$$

Let $g$ be a function as in Lemma 1 ; to prove that $g=0$, it is enough to show that $\lim _{r \rightarrow 1^{-}} \int_{0}^{2 \pi} \log \left|g\left(r e^{i \theta}\right)\right| d \theta=-\infty$ [Ga, proof of Th. 4.1, p. 65]. We will prove that $|g|$ is small enough, often enough, on circles of radii tending to 1.

Set

$$
s_{n}:=\sum_{k \leq p_{n}}\left(1-\left|a_{n, k}\right|^{2}\right)\left|g\left(a_{n, k}\right)\right|=2^{-n} \sum_{k \leq p_{n}}\left|g\left(a_{n, k}\right)\right| .
$$

Since $\sum_{n} s_{n}<\infty, \lim _{n \rightarrow \infty} s_{n}=0$. Let

$$
A_{n}=\left\{k: 1 \leq k \leq p_{n},\left|g\left(a_{n, k}\right)\right| \geq \gamma_{n}\right\} .
$$

By Chebyshev's inequality, \# $\# A_{n} \leq 2^{n} s_{n} / \gamma_{n} \leq p_{n} / 2$ for $n$ large. Thus, if we set $A_{n}^{\prime}:=\left\{1, \ldots, p_{n}\right\} \backslash A_{n}, \# A_{n}^{\prime} \geq p_{n} / 2$.

Set $J\left(a_{n, k}\right):=\left(\left(k-\frac{1}{2}\right) \ell_{n},\left(k+\frac{1}{2}\right) \ell_{n}\right)$, an interval on the real line which we may think of as an arc on the unit circle. Then

$$
\lambda_{1}\left(\bigcup_{k \in A_{n}^{\prime}} J\left(a_{n, k}\right)\right) \geq C p_{n} \ell_{n}
$$

Since $g \in H^{\infty}(\mathbb{D}),\left|g^{\prime}(z)\right| \leq C\left(1-|z|^{2}\right)^{-1}$, so that for $\theta \in J\left(a_{n, k}\right)$,

$$
\left|g\left(\left(1-2^{-n}\right)^{\frac{1}{2}} e^{i \theta}\right)-g\left(a_{n, k}\right)\right| \leq C 2^{n} \ell_{n} ;
$$

thus when $k \in A_{n}^{\prime}$,

$$
\log \left|g\left(\left(1-2^{-n}\right)^{\frac{1}{2}} e^{i \theta}\right)\right| \leq \log \left(\gamma_{n}+C 2^{n} \ell_{n}\right) \leq C+\log \gamma_{n} .
$$


The integral is now estimated by $p_{n} \ell_{n} \log \gamma_{n} \leq-C\left|\log \gamma_{n}\right|^{1 / 2} \rightarrow-\infty$, and the arc subtended by the points $a_{n, k}$ for a fixed $n$ is of length $p_{n} \ell_{n}=\left|\log \gamma_{n}\right|^{-1 / 2} \rightarrow 0$ as $n \rightarrow \infty$, so $\left\{a_{k}\right\}$ only accumulates at 1 .

\section{ACKNOWLEDGEMENTS}

This paper arose as a by-product of my collaboration with Xavier Massaneda, with whom it was much discussed. The key observation for the proof of Proposition 3 is due to Bo Berndtsson, who also started me wondering about sampling in the Hardy spaces. Also I should thank Alex Heinis for bringing [Br-Sh-Ze] to my attention (while he was studying under Jan Wiegerinck's direction). At the excellent workshop on Bergman spaces and related topics organized by Kristian Seip in Trondheim from September 29 to October 2, 1996, I had useful discussions with Carl Sundberg and other mathematicians too numerous to mention.

\section{REFERENCES}

[Br-Ni-Øy] Bruna J., Nicolau A., Øyma K., A note on interpolation in the Hardy spaces of the unit disc, Proc. Amer. Math. Soc. 124 (1996), 1197-1204. MR 96g:30066

[Br-Sh-Ze] Brown L., Shields A., Zeller K., On absolutely convergent exponential sums, Trans. Amer. Math. Soc. 96 (1960), 162-183. MR 26:332

[Du] Duren P., Theory of $H^{p}$ Spaces, Academic Press, New York, 1970. MR 42:3552

[Ga] Garnett J., Bounded analytic functions, Academic Press, New York, $1981 . \quad$ MR 83g:30037

[La] Landau H.J., Necessary density conditions for sampling and interpolation of certain entire functions, Acta Math. 117 (1967), 37-52. MR 36:5604

[Lu] Luecking D., Dominating measures for spaces of analytic functions, Ill. J. Math. 32 (1988), 23-39. MR 89e:46060

[Ly-Se] Lyubarskii Yu., Seip K., A uniqueness theorem for bounded analytic functions, Bull. London Math. Soc. 29 (1997), 49-52. MR 97m:30037

[Se1] Seip K., Beurling type density theorems in the unit disk, Invent. Math. 113 (1993), 21-39. MR 94g:30033

[Se2] Seip K., Regular sets of sampling and interpolation for weighted Bergman spaces, Proc. Amer. Math. Soc. 117 (1993), 213-220. MR 93c:30051

[Sh-Sh] Shapiro H. S., Shields A., On some interpolation problems for analytic functions, Amer. J. Math. 83 (1961), 513-532. MR 24:A3280

[St] Stein E. M., Singular integrals and differentiability properties of functions, Princeton University Press, Princeton, 1970. MR 44:7280

Laboratoire Emile Picard, Université Paul Sabatier, 118 route de Narbonne, 31062 Toulouse Cedex, France

E-mail address: pthomas@cict.fr 